\title{
Arterias Hepáticas Aberrantes. Estudio en 64 Cadáveres Disecados
}

\author{
Aberrants Hepatic Arteries. Research over 64 Disections \\ Ottone, Nicolás Ernesto; Arrotea Molina, Agustín; Domínguez, Mario Luis; Lo Tartaro, Maximiliano; \\ García de Quiros, Nicolás; Medan, Carlos; Blasi, Esteban \& Bertone, Vicente Hugo
}

OTTONE, N. E.; ARROTEA MOLINA, A.; DOMÍNGUEZ, M. L.; LO TARTARO, M.; GARCIA DE QUIROS, N.; MEDAN, C. BLASI, E. \& BERTONE, V. H. Arterias hepáticas aberrantes. Estudio en 64 cadáveres disecados. Int. J. Morphol., 24(4):581-585, 2006.

RESUMEN: La promoción del conocimiento de la distribución vascular hepática, tanto clásica como de sus variaciones, es fundamental para planear y realizar todos los procedimientos quirúrgicos y radiológicos en el abdomen superior.

Se disecaron 64 cadáveres, formolizados al 10\%, entre 1980 y 2005. Primero se abrió la cavidad abdominal con incisión en boca de horno, luego se investigaron fundamentalmente el omento menor y el pedículo hepático y se procedió a la disección del compartimiento supramesocólico.

Los resultados de las disecciones fueron agrupados en 5 clases: Clase 1 (73,44\%): Disposición arterial clásica. Clase 2A (9,37\%): Arterias hepáticas izquierdas aberrantes accesorias con origen en la arteria gástrica izquierda. Clase 2B (4,69\%): Arterias hepáticas izquierdas aberrantes reemplazantes con origen en la arteria gástrica izquierda. Clase 3A (3,12\%): Arterias hepáticas derechas aberrantes accesorias naciendo con origen en la arteria mesentérica superior. Clase 3B (3,12\%): Arterias hepáticas derechas aberrantes reemplazantes con origen en la arteria mesentérica superior. Clase 4 (3,12\%): Disposición combinada de arterias hepáticas izquierda y derecha aberrantes. Clase 5 (3,12\%): Arteria hepática común aberrante reemplazante con origen en la arteria mesentérica superior.

Concluimos que la disposición normal de la anatomía hepática fue hallada en el 73,44\% y que existe una significativa frecuencia de aparición de arterias hepáticas aberrantes (26,56\%). De esta manera, definimos dos sitios fundamentales de origen de arterias hepáticas aberrantes: la arteria gástrica izquierda de la que se originan las arterias hepáticas izquierdas aberrantes (Clase 2 (A-B) 14,06\%), recorriendo el omento menor y la arteria mesentérica superior de la que se originan las arterias hepáticas derechas aberrantes (Clase 3 (A-B) 6,24\%), realizando un peligroso trayecto retroduodenoportal. Es fundamental la investigación precisa del omento menor y de la cara posterior del pedículo hepático por la posibilidad de hallazgo de estas arterias hepáticas aberrantes.

PALABRAS CLAVE: Hígado; Arteria hepática; Arterias hepáticas aberrantes; Hilio hepático.

\section{INTRODUCCIÓN}

El interés por el conocimiento de la anatomía de la arteria hepática y sus variaciones no es nuevo, ya que ha sido investigado adecuadamente desde tiempos antiguos. Uno de los primeros investigadores fue Haller en 1756 quien incluyó las variaciones del tronco celíaco. Tidemann en 1822 (in Michels, 1953) describió múltiples anomalías y Adachi en 1928 clasificó estas variaciones arteriales en 28 subgrupos. También encontramos los análisis clásicos realizados por Flint en 1923, Michels (1955) con su trabajo en 200 cadáveres y Vandamme et al. en 1969. Estudios más recientes realizados por Hiatt et al. (1994) en 1000 casos y la revisión de las variaciones hepáticas a través de angiografia digital en 600 pacientes realizadas por Covey et al. (2002), entre otros trabajos, resaltan la importancia de estas variaciones arteriales.

En la actualidad, un nuevo empuje recibió el estudio anatómico de la arteria hepática, con la introducción del transplante de hígado. Los patrones arteriales son importantes para planear y realizar todos los procedimientos quirúrgicos y radiológicos en el abdomen superior, incluyendo las cirugías laparoscópicas de las vías biliares (del Sol et al., 2001.) 
El objetivo de este trabajo fue establecer la frecuencia de hallazgo de las arterias hepáticas aberrantes y la importancia del conocimiento de su existencia para su preservación posterior, en el momento de afrontar los procedimientos radiológicos y quirúrgicos en el abdomen superior con seguridad.

\section{MATERIAL Y MÉTODO}

En el Equipo de Disección de la 2a Cátedra de Anatomía de la Facultad de Medicina de la Universidad de Buenos Aires, Argentina, se realizó entre 1980 y 2005, la disección de 64 cadáveres formolizados al 10\%. En ellos se operó la región abdominal disecándose la región supra-mesocólica, buscando variaciones de la distribución vascular de toda esta región, centrándonos en el hallazgo de arterias hepáticas aberrantes.

\section{RESULTADOS}

Consideramos oportuno, en primer lugar, definir los términos "aberrante", "accesoria" y "reemplazante". El término "aberrante" se aplica a aquellas arterias hepáticas que aparecen en forma variable, originándose de un sitio distinto al del vaso normal (por ejemplo: aorta, arterias mesentérica superior, mesentérica inferior, etc.), que clásicamente no se encuentran en la región estudiada. A las arterias hepáticas aberrantes se las dividió en dos tipos: "accesoria" y "reemplazante". Las arterias hepáticas aberrantes accesorias son aquellas que, originándose de un sitio distinto al normal, aparecen junto con el vaso de presencia clásica y se suman con éste a la vascularización de un sector determinado del hígado. Mien- tras que las arterias hepáticas aberrantes reemplazantes son aquellas que se encuentran presentes en ausencia del vaso de disposición normal, originándose de un sitio distinto al del vaso normal, y lo reemplaza en su función, encargándose por sí solo de la vascularización de determinado sector hepático.

A partir de las estadísticas obtenidas, desarrollamos una clasificación basada en las siguientes 5 clases de disposición arterial (Tabla I):

Clase 1 (Normal). Disposición normal de las arterias hepáticas; la AHC se origina del TC y posteriormente se divide en AHP y AG, finalmente la AHP se divide luego de un corto trayecto en AHD y AHI.

Clase 2A (AHIAacc de AGI). Arteria hepática izquierda accesoria con origen en la arteria gástrica izquierda.

Clase 2B (AHIAreemp de AGI). Arteria hepática izquierda reemplazante con origen en la arteria gástrica izquierda.

Clase 3A (AHDAacc de AMS). Arteria hepática derecha accesoria con origen en la arteria mesentérica superior.

Clase 3B (AHDAreemp de AMS). Arteria hepática derecha reemplazante con origen en la arteria mesentérica superior.

Clase 4 (AHIAacc de AGI + AHDAreemp de AMS o viceversa). Esta disposición abarca la posibilidad de aparición de múltiples ramas aberrantes, tanto accesorias como reemplazantes, de las arterias hepáticas derecha e izquierda.

Clase 5 (AHCAreemp de AMS). La arteria hepática común originándose directamente de la arteria mesentérica superior.

Tabla I. Disposición de las arterias hepáticas en 64 cadáveres disecados

\begin{tabular}{|c|c|c|c|}
\hline Clase & Detalle & Cantidad & Porcentaje \\
\hline 1 & Disposición normal - "clásica" & 47 & 73,44 \\
\hline $2 \mathrm{~A}$ & \multirow{4}{*}{$\begin{array}{l}\text { AHÍAacc. naciendo de AGI } \\
\text { AHÍAreemp. naciendo de AGI } \\
\text { AHDAacc. naciendo de AMS } \\
\text { AHDAreemp. naciendo de } \\
\text { AMS }\end{array}$} & 6 & 9,37 \\
\hline $2 \mathrm{~B}$ & & 3 & 4,69 \\
\hline $3 \mathrm{~A}$ & & 2 & 3,12 \\
\hline $3 \mathrm{~B}$ & & 2 & 3,12 \\
\hline 4 & $\begin{array}{l}\text { AHIAacc. de AGI + } \\
\text { AHDAreemp. de AMS o } \\
\text { AHIAreemp. de AGI + } \\
\text { AHDAacc. de AMS }\end{array}$ & $2^{1,2}$ & 3,12 \\
\hline 5 & AHCAreemp. de AMS & 2 & 3,12 \\
\hline
\end{tabular}

${ }^{1}$ Se encontró un tipo de cada combinación de variedad arterial.

${ }^{2}$ La disposición AHIAacc. de AGI + AHDAreemp. de AMS presenta la AHDAreemp. originándose de la AMS, pero ésta a su vez, de un tronco celíaco-mesentérico.

AHIAacc: arteria hepática izquierda aberrante accesoria; AHIAreemp: arteria hepática izquierda aberrante remplazante; AHDAacc: arteria hepática derecha aberrante accesoria; AHDAreemp: arteria hepática derecha aberrante reemplazante; AHC: arteria hepática común; AHCAreemp: arteria hepática común aberrante reemplazante; AGI: arteria gástrica izquierda; AMS: arteria mesentérica superior. 
Tabla II. Comparación de los resultados del estudio del origen de las aa. hepáticas con los obtenidos por otros invest igadores

\begin{tabular}{lccccccc}
\hline $\begin{array}{l}\text { Variación } \\
\text { anatómica }\end{array}$ & $\begin{array}{c}\text { EDSCA } \\
(\mathbf{n = 6 4 )}\end{array}$ & $\begin{array}{c}\text { Hiatt } \\
(\mathrm{n}=1000)\end{array}$ & $\begin{array}{c}\text { Covey } \\
(\mathrm{n}=600)\end{array}$ & $\begin{array}{c}\text { Michels } \\
(\mathrm{n}=200)\end{array}$ & $\begin{array}{c}\text { Varotti } \\
(\mathrm{n}=96)\end{array}$ & $\begin{array}{c}\text { Hardy } \\
(\mathrm{n}=70)\end{array}$ & $\begin{array}{c}\text { Estandarización } \\
(\mathrm{n}=2030)\end{array}$ \\
\hline Clase 1 (\%) & $\mathbf{7 3 , 4}$ & 75,7 & 61,3 & 55,0 & 70,8 & 61,5 & 66,3 \\
\hline Clase 2 (A-B)(\%) & $\mathbf{1 4 , 1}$ & 9,7 & 14,5 & 18,0 & 12,5 & 7,1 & 12,7 \\
\hline Clase 3 (A-B)(\%) & $\mathbf{6 , 2}$ & 10,6 & 10,2 & 18,0 & 13,5 & 10,0 & 11,5 \\
\hline Clase 4 (\%) & $\mathbf{3 , 1}$ & 2,3 & 4,5 & 4,0 & 2,1 & 7,1 & 3,8 \\
\hline Clase 5 (\%) & $\mathbf{3 , 1}$ & 1,5 & 2,0 & 2,5 & 1,0 & 4,2 & 2,4 \\
\hline Otros (\%) & $\mathbf{0 , 0}$ & 0,2 & 7,5 & 0,5 & 0,0 & 10,0 & 3,0 \\
\hline
\end{tabular}

La columna de estandarización se construyó promediando los porcentajes de cada una de las clases de variaciones anatómicas encontradas por los distintos investigadores, llegando a un porcentaje común.

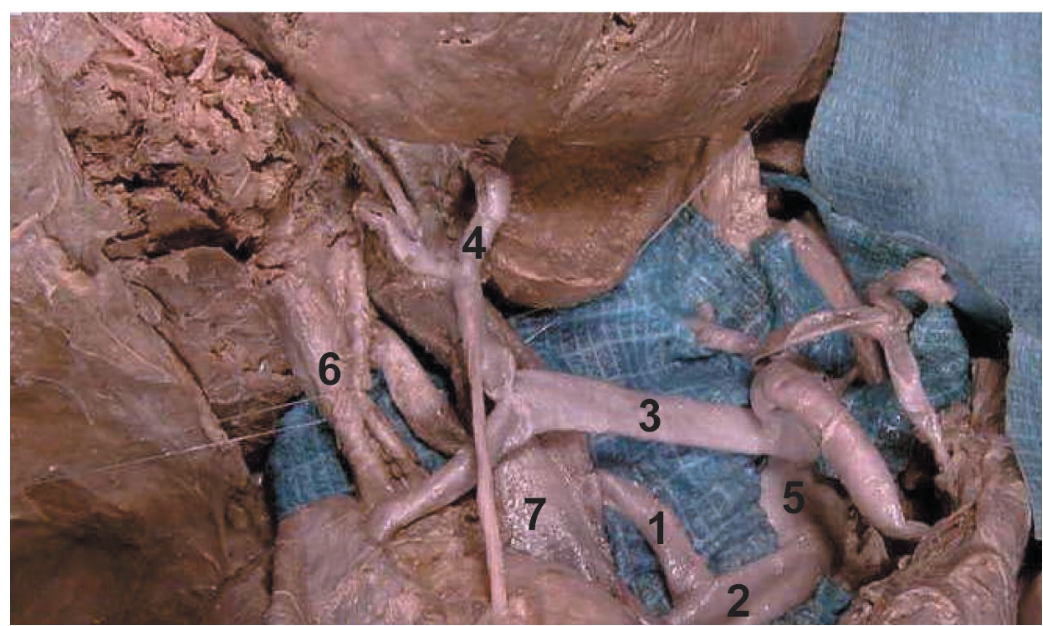

Fig. 1. Disposición Clase 3A de las arterias hepáticas. Presencia de una arteria hepática derecha aberrante reemplazante (1) originándose de la arteria mesentérica superior (2). Tanto el duodeno como el páncreas fueron reclinados hacia abajo para poder apreciar el peligroso trayecto retroduodenoportal. Arteria hepática común (3), Arteria hepática izquierda (4); Aorta (5); Conducto hepático común (6); Vena porta (7).

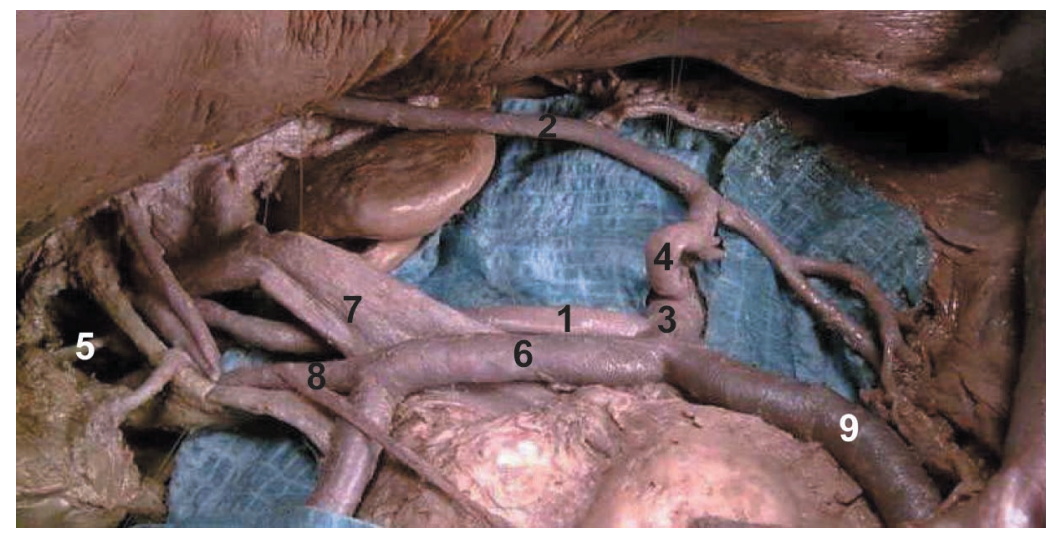

Fig. 2. Disposición Clase 4 de las arterias hepáticas. Presencia de una arteria hepática izquierda aberrante reemplazante (2), originándose de la arteria gástrica izquierda (4) junto a una arteria hepática derecha aberrante accesoria (1) con origen en el tronco celíaco (3). Arteria cística (5); Arteria hepática común (6); Vena porta (7); Arteria hepática derecha (8); Arteria esplénica (9).

Observamos que en 17 de los 64 cadáveres disecados (26,56\%), presentaban arterias hepáticas aberrantes. De éstas, en 9 casos $(57,14 \%)$ encontramos arterias hepáticas izquierdas aberrantes; en 4 casos $(21,43 \%)$ arterias hepáticas derechas aberrantes; en dos casos (14,28\%) arterias hepáti- cas comunes aberrantes, y en dos casos $(14,28 \%)$ encontramos, al mismo tiempo, una arteria hepática izquierda aberrante accesoria junto con una arteria hepática derecha aberrante reemplazante y viceversa.

Con respecto a las arterias hepáticas izquierdas aberrantes, un 66,67\% de ellas eran accesorias, mientras que el 33,33\% restante eran arterias hepáticas izquierdas reemplazantes. Casiragui (1982), considera a esta variación, ya sea accesoria o reemplazante, una persistencia embriológica de la rama hepática de la arteria gastrohepática). Con respecto a las arterias hepáticas derechas aberrantes, en un $50 \%$ de los casos correpondían a arterias hepáticas derechas reemplazantes y el 50\% restante a la variedad accesoria.

El lugar de origen más común de las arterias hepáticas izquierdas aberrantes corresponde, tanto de las variedades accesoria y reemplazante, a la arteria gástrica izquierda (naciendo sólo una de la arteria hepática común). Con respecto a las arterias hepáticas derechas aberrantes, tanto accesorias como reemplazantes, todas ellas se originan de la arteria mesentérica superior.

En la mitad de los casos, las arterias hepáticas derechas aberrantes, correspondían a la variedad reemplazante, mientras que sólo la tercera parte de arterias hepáticas izquierdas aberrantes eran reemplazantes. Apesar que lo más común, según nuestra investigación, sea encontrar arterias hepáticas izquierdas aberrantes accesorias, dentro de las arterias hepáticas derechas aberrantes, existe un $50 \%$ de probabilidades de que sean reemplazantes, originándose directamente de la arteria mesentérica superior. 


\section{DISCUSIÓN}

La disposición normal de las arterias hepáticas (Clase I) se presentó en el $73 \%$ de nuestros casos, muy similar a lo observado por Hiatt (1994) de $75,7 \%$ y por Varotti (2004) de $70,8 \%$. Sin embargo, otros autores como Hardy \& Jones, (2001) y Covey et al., establecen valores inferiores. Si promediamos los resultados de todos los estudios, el porcentaje normal sería de alrededor del 66,3\%.

Cuando es necesario desarrollar la gastrectomía radical en el cáncer de estómago (Suzuki et al., 1971), el conocimiento de las arterias hepáticas aberrantes es importante. Existe la posibilidad que la arteria hepática izquierda esté originándose de la arteria gástrica izquierda (Clases 2AB). Cuando la arteria gástrica izquierda es ligada a nivel de su origen, durante la gastrectomía subtotal radical del carcinoma, existirá una alta incidencia de necrosis del lóbulo izquierdo del hígado, si no es reconocida esta variación durante la operación (Redman, 1969). La arteria hepática derecha se origina muy frecuentemente de la arteria mesentérica superior (Clases 3A-B). En este tipo de variación, la arteria corre entre la vena cava inferior y la vena porta antes de aparecer a nivel del trígono cistohepático. Mucha atención debe tenerse con esta arteria en la colecistectomía, como así también durante la pancreatectomía.

Con respecto al transplante hepático, las variaciones de la arteria hepática, si bien no causan contraindicaciones absolutas, se hace necesario estar bien informado de estas variaciones, ya que es un factor determinante para la simplificación de la cirugía, disminuyendo los efectos colaterales y permitiendo desarrollar una cirugía con éxito (Lee, 2003). La angiografía por TC es un elemento fundamental para el hallazgo de las variaciones de la arteria hepática, aliado imprescindible al momento de afrontar la cirugía hepatobiliar y de transplante en forma exitosa (Suzuki et al., 1971).

Dos son los lugares y las variaciones que fundamentalmente es necesario reconocer al momento de trabajar en la región supramesocólica, debido a la alta frecuencia de aparición de arterias hepáticas aberrantes $(26,56 \%)$ y a las consecuencias que una lesión podría provocar. Por un lado, se debe observar cuidadosamente el omento gastrohepático y determinar la presencia de una arteria hepática izquierda aberrante, que puede originarse de la arteria gástrica izquierda, y recorrer este omento en búsqueda del hilio hepático. Por el otro lado, es necesario examinar la cara posterior del pedículo hepático y observar la eventual presencia de una arteria hepática derecha aberrante, la cual nace de la arteria mesentérica superior, desarrollando un peligroso recorrido retroduodenoportal para alcanzar también el hilio hepático, lo que supone un alto riesgo de lesión, en aquellos que efectúen la colecistectomía e ignoren el alto porcentaje de aparición de la misma.

AGRADECIMIENTOS. A los Drs. V. Hugo Bertone, Carlos Medan y Esteban Blasi por la confianza, apoyo y consejo que dieron para la realización de este trabajo. Al Dr. Mariano del Sol por su desinteresado e importante consejo y colaboración para la publicación de este trabajo.

OTTONE, N. E.; ARROTEA MOLINA, A.; DOMÍNGUEZ, M. L.; LO TARTARO, M.; GARCIA DE QUIROS, N.; MEDAN, C. BLASI, E. \& BERTONE, V. H. Aberrants hepatic arteries. Research over 64 disections. Int. J. Morphol., 24(4):581-585, 2006.

SUMMARY: The promotion of the knowledge of the classic hepatic vascular distribution and its variations is fundamental to plan and to make all the surgical and radiological procedures in the superior abdomen.

64 corpses preserved with formol to $40 \%$ were dissected, between 1980 and 2005 . First the abdominal cavity with incision in furnace mouth was opened, soon the gastrohepatic epiplon and the hepatic pedicle were investigated, and finally it was dissected all the supramesocolic sector.

The results of the dissection were grouped in 5 classes: Class $1(73,44 \%)$ : Classic arterial disposition. Class 2A(9,37\%): Accessory aberrant left hepatic artery being born of the left gastric artery. Class 2B (4,69\%): Replacing aberrant left hepatic artery being born of the left gastric artery. Class 3A (3,12\%): Accessory aberrant right hepatic artery being born of the superior mesenteric artery. Class 3B (3,12\%): Replacing aberrant right hepatic artery being born of the superior mesenteric artery. Class 4 (3,12\%): Combined disposition of aberrants hepatics arteries, right and left. Class 5 (3,12\%): Replacing aberrant common hepatic artery being born of the superior mesenteric artery.

We concluded that the normal disposition of the hepatic anatomy was found in $73,44 \%$. The aberrants hepatics arteries were found with significant, $26,56 \%$. This way, we defined two fundamental sites of birth of aberrant hepatics arteries: the left gastric artery is the site of birth of the aberrants lefts hepatics arteries (Class 2 (A-B) 14.06\%), crossing gastrohepatic epiplon, and the superior mesenteric artery site of birth of the aberrant rights hepatics arteries (Class 3 (A-B) 6.24\%), making a dangerous retroduodenoportal passage. For that reason the precise investigation of gastrohepatic epiplon and the back of the hepatic pedicle by the possibility of finding of these aberrant hepáticas arteries is fundamental.

KEY WORDS: Liver; Hepatic artery; Aberrants hepatics arteries ;Hepatic hilium. 


\section{REFERENCIAS BIBLIOGRÁFICAS}

Adachi, B. Das arteriensystem der Japaner. Kyoto, Universität zu Kyoto, 1928. V.2.

Bertone, V. H.; Fernández Russo, G. A. H. \& Calderaro, M. S. Documentación de las variaciones anatómicas halladas durante las disecciones de rutina (Principios organizativos y metodología empleada). Universidad de Buenos Aires, Facultad de Medicina, $3^{\text {a }}$ Cátedra de Anatomía. XIX Congreso de la Asociación Rioplatense de Anatomía, 1982.

Casiragui, J. C. et al. Anatomía del cuerpo humano, funcional y quirúrgica. Regiones del Abdomen y de la Pelvis. Editorial Alboazul, Montevideo. 1982. V. 4.

Covey, A. M.; Brody, L. A.; Maluccio, M. A.; Getrajdman, G. I. \& Brown, K. T, Variant Hepatic Arterial Anatomy Revisited: Digital Subtraction Angiography Performed in 600 Patients. Radiology, 224:542-7, 2002.

Del Sol, M.; Olave, E. \& Epulef, V. Variación anatómica de las arterias hepática propia y pancreaticoduodenal inferior. Aspectos anatomo-clínicos. Rev. Chil. Cs. Méd. Biol., 11(2):51-54, 2001.

Flint, E. R. Abnormalities of the right hepatic, cistyc and gastroduodenal arteries, and of the bile ducts. Brit. $J$. Surg., 10: 509, 1923.

Haller, A. 1756 apud Vandamme, J. P. L. \& Bonte, J. op cit. The branches of the celiac trunk. Acta Anat., 122:1104, 1985 .

Hardy, K. J. \& Jones, R. M. The hepatic artery: A reminder of surgical anatomy. J. Coll. Surg. Edinb., 46(3):16870,2001 .

Hiatt, J. R.; Gabbay, J. \& Busuttil, R. W. Surgical anatomy of the hepatic arteries in 1000 cases. Ann. Surg., 220(1):50-2, 1994.

Lee, S. S.; Kim. T. K.; Byun, J. H.; Ha, H. K.; Kim. P. N.; Kim. A. Y.; Lee, S. G. \& Lee, M. G. Hepatic arteries in potential donors for living related liver transplantation: Evaluation eith multi-detector row ct angiography. Radiology, 227(2):391-9, 2003.

Michels, N. A. Variational anatomy of the hepatic, cystic, and retroduodenal arteries; a statistical analysis of their origin, distribution, and relations to the biliary ducts in two hundred bodies. AMA Arch Surg., 66(1):20-34, 1953.

Redman, H. C. \& Reuter, S. R. Angiographic demonstration of surgically important vascular variations. Surg. Gynecol. Obstet., 129:33-9, 1969.

Suzuki, T.; Nakayasu, A.; Kawabe, K. et al. Surgical significance of anatomic variations of the hepatic artery. Am. J. Surg., 122:505-12, 1971.

Tidemann, 1822 apud Michels, N. A. Variational anatomy of the hepatic, cystic, and retroduodenal arteries; a statistical analysis of their origin, distribution, and relations to the biliary ducts in two hundred bodies. $A M A$ Arch Surg., 66(1):20-34, 1953. op cit.

Vandamme, J. P. J.; Bonte, J. \& Schueren, G. A revaluation of hepatic and cystic arteries. The importance of the aberrant hepatic branches. Acta Anat., 73:192-209, 1969.

Varotti, G.; Gondolesi, G. E.; Goldman, J.; Wayne, M.; Florman, S. S.; Schwartz, M. E.; Miller, C. M. \& Sukru, E. Anatomic variations in right liver living donors. $J$. Am. Coll. Surg., 198(4):577-82, 2004.

Dirección para correspondencia:

Nicolas $\mathcal{E}$. Ottone

$\mathcal{A v}$. Honorio Pueyrredon 737 3er. piso

Codigo Postal 1405

Ciudad Autónoma de Buenos Aires

ARGE $\mathcal{N}$ TINA

Email:nicolas_ottone@yahoo.com

Received : 25-07-2006

Accepted: 17-11-2006 
